\title{
Novel Coronavirus-Induced Right Ventricular Failure and Point of Care Echocardiography: A Case Report
}

\author{
Lu Chen ${ }^{a, e}$ Gautham Upadhya ${ }^{b}$ Uta S. Guo ${ }^{c}$ Pooja Belligund ${ }^{d}$ David K. Lee ${ }^{d}$ \\ Isaac Shalom ${ }^{d}$ Gangacharan R. Dubey ${ }^{d}$ Mohammad R. Al-Ajam ${ }^{\text {d }}$ \\ Cristina A. Mitre ${ }^{\mathrm{e}}$ \\ ${ }^{a}$ Department of Internal Medicine, Downstate Medical Center, State University of New York, Brooklyn, NY, USA; \\ ${ }^{b}$ Division of Cardiology, Department of Internal Medicine, Downstate Medical Center, State University of New York, \\ Brooklyn, NY, USA; 'Sunrise Health Consortium, Southern Hills Hospital Family Medicine GME, Las Vegas, NV, USA; \\ ${ }^{\mathrm{d}}$ Division of Pulmonary and Critical Care Medicine, Department of Internal Medicine, Brooklyn Campus, Veterans \\ Affairs New York Harbor Healthcare System, Brooklyn, NY, USA; ${ }^{e}$ Division of Cardiology, Department of Internal \\ Medicine, Brooklyn Campus, Veterans Affairs New York Harbor Healthcare System, Brooklyn, NY, USA
}

\section{Established Facts}

- Novel coronavirus disease 2019 exhibits different patterns of cardiac injury, including acute coronary syndrome, malignant arrhythmia, myocarditis, pericarditis, and heart failure.

- Point of care ultrasound is being increasingly utilized for quick assessment of cardiac and pulmonary function at the bedside.

\section{Novel Insights}

- With the limited availability of personal protective equipment, bedside point of care ultrasound can minimize staff exposure and ration available personal protective equipment.

- Serial point of care ultrasound in coronavirus disease 2019 patients can help assess both pulmonary and cardiac function and guide further clinical therapy.

\section{Keywords}

Cor pulmonale · Point of care echocardiography · Novel coronavirus · Coronavirus disease 2019

\section{Abstract \\ Various cardiovascular complications have been reported in patients with coronavirus disease 2019. Common complica-}

tions include acute myocardial injury, myocarditis, arrhythmia, pericarditis, heart failure, and shock. We present a case of cor pulmonale diagnosed with serial point of care ultrasound. Given the current shortage of personal protective equipment (PPE) and high infectivity of this virus, we acknowledge the utility of this tool in obtaining important clinical information while minimizing exposure and PPE consumption.

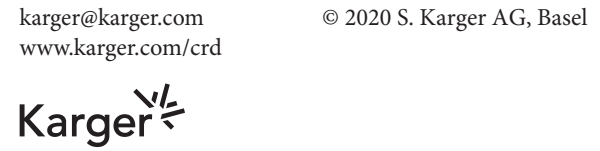




\section{Introduction}

The ongoing coronavirus disease 2019 (COVID-19) caused by severe acute respiratory syndrome coronavirus 2 (SARS-CoV-2) has resulted in over 300,000 cases in the USA and has claimed the lives of over 10,000 Americans by early April 2020 [1]. Moreover, the incidence continues to rise daily in our country. The understanding of COVID-19 is evolving but is still limited to date. Cardiovascular complications of COVID-19 have been reported in anywhere between 7 and 20\% of patients [2-5]. More importantly, the presence of cardiovascular complications was described as an independent risk factor for increased critical illness as well as mortality in COVID-19 patients $[3,4]$. Various cardiovascular insults have been reported, including acute myocardial injury (defined as high-sensitivity troponin serum levels $>99$ th percentile upper reference limit), myocarditis, arrhythmia, and shock [2, 6-8]. Unfortunately, differentiation of the types of shock have not been reported thus far. Some of the medications used to treat COVID-19, none of which is based on randomized controlled human trials, are also known to increase QT interval and potentially precipitate sudden cardiac death [9].

Furthermore, due to the highly contagious and widespread nature of the COVID-19 pandemic, a shortage of personal protective equipment (PPE) has become apparent [10]. It has driven a reconsideration of the utilization of diagnostic tools and therapeutic approaches [11]. Point of care ultrasound (POCUS) is emerging as a powerful tool to quickly assess critically ill patients [12]. POCUS requires a single operator, most frequently the intensivist and eliminates the need to transport patients. POCUS can potentially limit health care worker (HCW) exposure and conserve available PPEs. Therefore, the purpose of this report is to describe the cardiovascular complication and to share our experience in utilizing POCUS in a critically ill COVID-19 patient.

\section{Case Report}

We present the case of a 73-year-old male with a history of diabetes mellitus, hypertension, hyperlipidemia, and gout. $\mathrm{He}$ presented to the emergency department due to progressive weakness and anorexia over the prior week. He was noted to be mildly hypoxic to $90 \%$ on pulse oximetry on admission, with increasing supplement oxygen requirement by a nasal cannula. His vital signs were otherwise stable with a body mass index of 24.8. Laboratory finding on admission was significant for leukopenia $(1,400 / \mu \mathrm{L})$, negative troponin I of $0.025 \mathrm{ng} / \mathrm{mL}$, and normal brain natriuretic peptide of $26 \mathrm{pg} / \mathrm{ml}$ (Table 1 ). His chest radiography showed subtle diffused bilateral interstitial opacities (Fig. 1a).

The patient rapidly progressed to severe hypoxic respiratory failure, requiring mechanical ventilation. Laboratory study at this time showed lymphopenia of $860 / \mathrm{cm}^{2}$. He was transferred to the intensive care unit for hemodynamic and ventilatory management. Acute respiratory distress syndrome (ARDS) ensued in the context of suspected COVID-19. He was started on broadspectrum antibiotics covering community-acquired pneumonia

Table 1. Laboratory studies of patient throughout hospitalization

\begin{tabular}{|c|c|c|c|c|c|c|c|c|c|c|}
\hline Measurements & Ref. values & \multicolumn{9}{|c|}{ Results by hospital days } \\
\hline $\mathrm{WBC}, \times 1,000 / \mu \mathrm{L}$ & $4-10.5$ & 1.4 & 2.2 & 2.3 & 2.1 & 2.2 & 5.9 & & 9.1 & \\
\hline \multicolumn{11}{|l|}{ Lymphocyte count } \\
\hline Relative, $\%$ & $20-40$ & & 39.8 & 16.7 & 21.7 & 13.8 & 10.4 & & 7.6 & \\
\hline Creatinine, $\mathrm{mg} / \mathrm{dL}$ & $0.4-1.2$ & 1.5 & 1.4 & 1 & 1 & 1.1 & 3.1 & & 4.3 & \\
\hline $\mathrm{AST}, \mathrm{U} / \mathrm{L}$ & $10-40$ & & 26 & 18 & 19 & 21 & 28 & 53 & 69 & \\
\hline $\mathrm{ALT}, \mathrm{U} / \mathrm{L}$ & $10-42$ & & 62 & 40 & 44 & 18 & 58 & 107 & 124 & \\
\hline $\mathrm{CRP}, \mathrm{mg} / \mathrm{dL}$ & $0-0.5$ & & & 26.6 & & 21.1 & 20.3 & 42.2 & & \\
\hline Troponin I, ng/mL & $0.006-0.06$ & 0.025 & & & & & 3.163 & 3.36 & 4.798 & 3.86 \\
\hline $\mathrm{BNP}, \mathrm{pg} / \mathrm{mL}$ & $0-100$ & 26 & & & & & & & & \\
\hline
\end{tabular}

WBC, white blood cells; AST, aspartate transaminase; ALT, alanine transaminase; CRP, C-reactive protein; BNP, brain natriuretic peptide. 
as well as norepinephrine and vasopressin for management of septic shock. Hydroxychloroquine and azithromycin were later added per prevailing recommendations of eminent health care centers.

Between hospital days 2 and 5 (HD2-5), the patient did not have escalation of vasopressor requirements. However, his respiratory status continued to gradually worsen. He required $\mathrm{FiO}_{2}$ of $60 \%$, and positive end-expiratory pressure was increased from 10 to $14 \mathrm{~mm} \mathrm{Hg}$. He maintained a plateau pressure between 26 and $28 \mathrm{~mm} \mathrm{Hg}$. POCUS on HD2 showed preserved systolic function of the left ventricle (LV) and a normal-sized right ventricle (RV). COVID-19 was confirmed on HD3. He developed pancytopenia by $\mathrm{HD} 5$, which was attributed to bone marrow suppression in the setting of systemic illness.

On HD6, the patient continued to deteriorate, requiring increased vasopressor support. Troponin I was noted to be elevated to $3.16 \mathrm{ng} / \mathrm{mL}$, and cardiology consultation was requested. POCUS at this time showed diffused b-lines of the overlaying lungs, a severely dilated RV, dilated and nonvariable inferior vena cava, and a hyperdynamic and underfilled LV with no apparent regional wall motion abnormalities (online suppl. video; see www.karger.com/doi/10.1159/000508527 for all online suppl. material). POCUS of the lower extremities showed fully compressible deep veins at the femoral and popliteal level bilat- erally. No significant ST segment abnormalities were seen on telemetry. The decision was made to provide transfusion of packed red blood cells to a target no less than $9 \mathrm{~g} / \mathrm{dL}$. Antiplatelet therapy as well as anticoagulation were held off because of decreasing hemoglobin level.

On HD7, the patient developed progressive renal failure and hepatic dysfunction with elevated transaminases. Telemetry showed ST segment elevation in the precordial lead but the troponin level minimally uptrended to $4.8 \mathrm{ng} / \mathrm{mL}$ over $24 \mathrm{~h}$. ST segment elevation was confirmed on electrocardiogram in the anterior and inferior leads compared to baseline (Fig. 2). Chest radiography showed progression of bilateral pulmonary opacities, and POCUS was unchanged (Fig. 1b-d). Interventional cardiology evaluation was requested, and the patient was deemed to be a poor candidate for invasive intervention and thrombolytic therapy. Two hours after the electrocardiogram showing ST segment elevation, repeated troponin downtrended to $3.9 \mathrm{ng} / \mathrm{mL}$. Given his progressive deterioration despite full supportive measures, a family discussion was held, and the decision was made for no resuscitation in case of cardiopulmonary arrest. The patient became progressively bradycardic and hypotensive. His telemetry tracing progressed to asystole and he expired on the night of HD7.

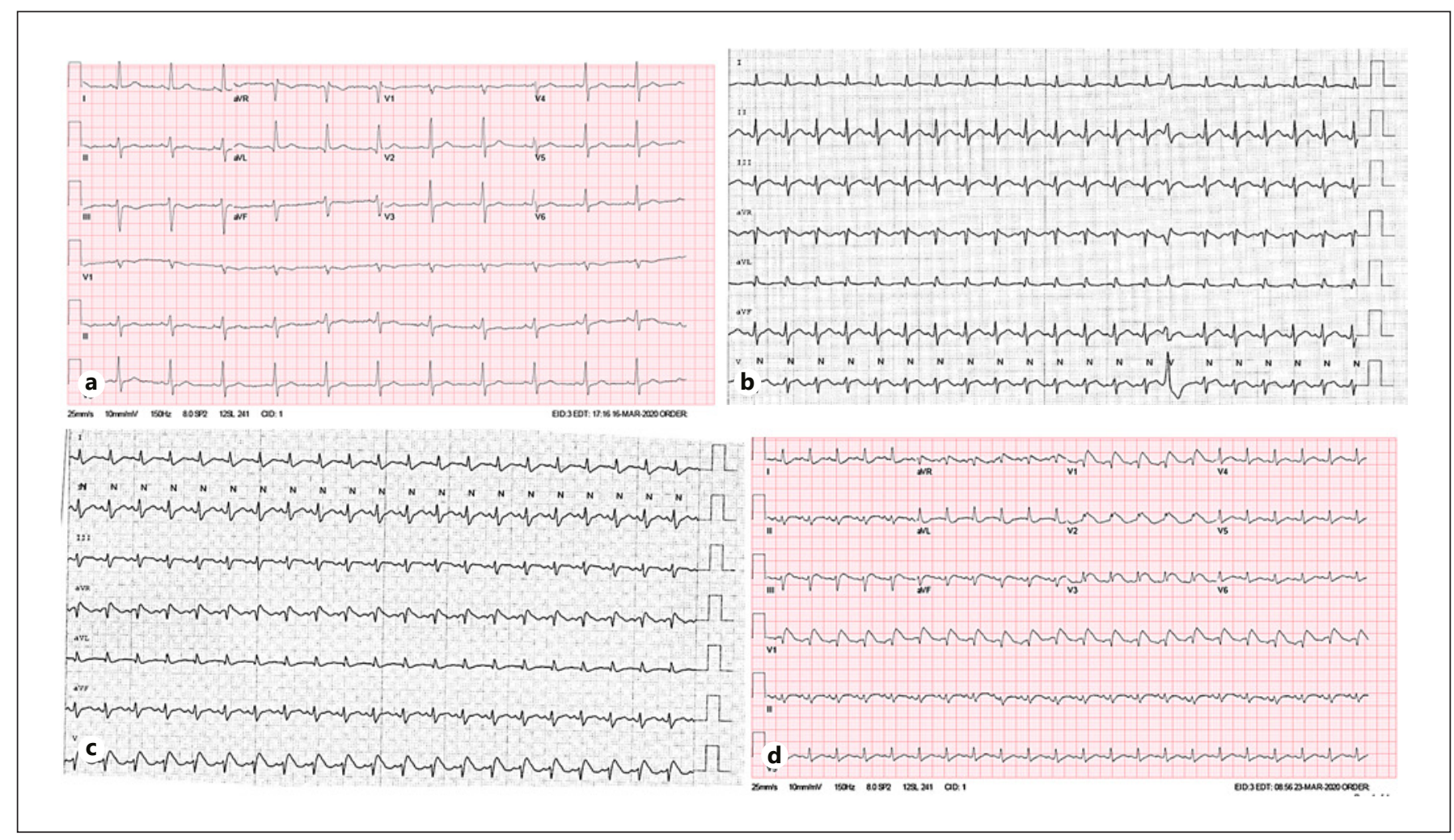

Fig. 1. a Electrocardiogram on admission showing normal sinus rhythm. b Telemetry tracing on morning of hospital day (HD) 6 without changes in ST segments. c Telemetry tracing on morning of HD7, showing ST segment elevation in precordial lead. $\mathbf{d}$ Electrocardiogram on HD7 showing ST segment elevation on anterior and inferior leads.

Novel Coronavirus-Induced RV Failure and Point of Care Echocardiography 


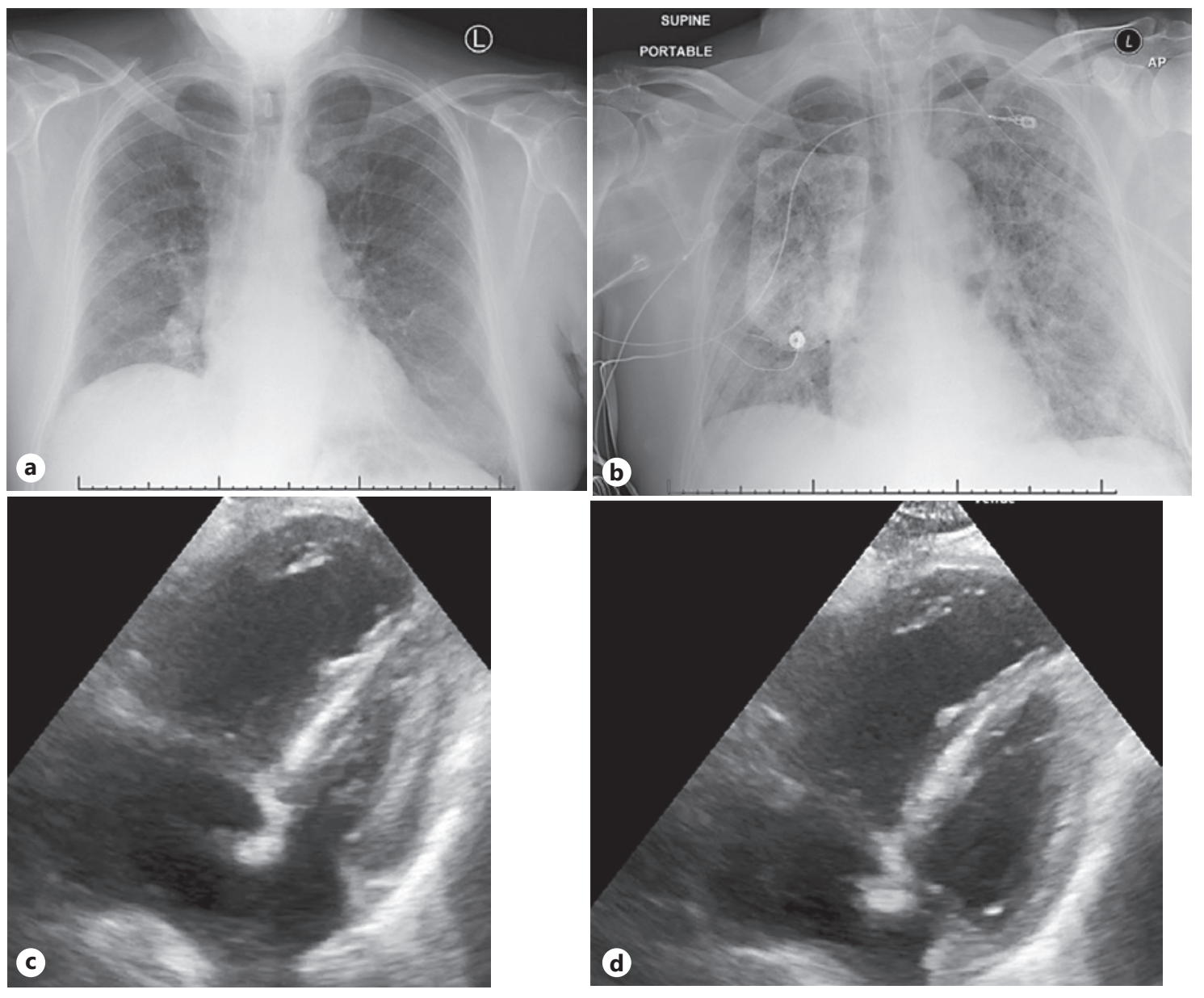

Fig. 2. a Admission chest radiography showing mild but diffused bilateral interstitial opacities. b Chest radiography on hospital day (HD) 7 showing worsening diffused interstitial opacities. Subcostal view of point of care echocardiography on HD7 in end systole (c) and end diastole (d), showing grossly dilated right ventricle and hyperdynamic left ventricle.

\section{Discussion}

The differential diagnosis for an elevated level of cardiac enzyme is wide. In the setting of COVID-19, in addition to noncardiac etiologies, specific cardiac causes of cardiac enzyme elevation to rule out should include myocarditis, pericarditis, myocardial infarction, heart failure, and arrhythmia [13]. Malignant arrhythmia was excluded upon review of available telemetry. Further differentiation of underlying cardiac etiology would be based on the finding of echocardiography. In the era of COVID-19, POCUS is emerging as a viable alternative to echocardiography to limit potential HCW exposure and ration limited PPEs [11, 12].

Due to a decreasing trend in troponin level, an acute coronary thrombotic event is unlikely, while myocarditis might have a role in observed electrocardiogram changes. Based on serial POCUS, this patient likely developed acute pulmonary hypertension in the setting of COVID-19, which resulted in progressive RV overload and cor pulmonale. His multiorgan dysfunction can be explained by obstructive shock in the setting of cor pulmonale, including congestive hepatopathy (transaminases) and cardiorenal syndrome manifested as acute kidney injury. The possibility of obstructive shock has been speculated by reports from China but has yet to be documented [14].

Near-normal plateau pressure observed on the ventilator in this patient as opposed to low compliance expected in patients with ARDS suggests an alternative etiology that resulted in acute pulmonary hypertension. Prior studies have suggested shortened thrombin time in critically ill COVID-19 patients [15], which raises the concern of pul- 
monary thromboembolism as well as intravascular microthrombosis in the pulmonary vasculature $[16,17]$. Our patient was treated with adequate venous thrombosis prophylaxis. He also lacked clinical and ultrasonographic (POCUS) signs suggestive of adequate venous thrombosis. With his progressive clinical course, microthrombosis in the pulmonary vasculature seems to be a possible explanation that resulted in his demise. Emerging evidence suggested that anticoagulation therapy in critically ill COVID patients has been associated with decreased mortality and should be considered in future cases [18]. Alternatively, pulmonary hypertension, likely due to progressive hypoxemia seen in ARDS has been recognized as early as 1977. However, pulmonary vasodilator therapy has not consistently shown improved survival in ARDS [19].

The use of POCUS in COVID-19 patients has been suggested to include advantages such as limiting exposure to HCWs, reducing nosocomial infection, and potentially lessening the burden of hospital admission [12]. In our experience, POCUS was utilized repeatedly in the care of this and other patients to provide quick assessments of the cardiovascular system. Furthermore, POCUS is able to provide timely evaluation of the pulmonary system that cannot be substituted with standard echocardiography or chest radiography. Given the current lack of PPE in New York, POCUS, reviewed by an experienced reading cardiologist, can potentially limit exposure for echocardiography and radiology technicians alike.

Based on the known cardiovascular complications in COVID-19 patients, point of care echocardiography can be used to differentiate potential myocarditis, acute coronary syndrome, and RV failure in patients in the critical care setting. Upon intubation and/or intensive care unit admission, POCUS should be obtained to establish baseline cardiac function, including estimation of left ventricular ejection fraction, systolic function, significant regional wall motion abnormalities, RV size and function. Upon clinical deterioration, especially in the setting of troponin elevation, reassessment of left ventricular ejection fraction, regional wall motion abnormalities, RV size and function is appropriate. In consultation with the cardiology team, serial POCUS can help differentiate common cardiac complications.

\section{Conclusion}

The understanding of COVID-19 is still evolving with the rising number of cases in the USA. Conserving available health care resources and protect HCWs is especial-

ly important at this critical time. POCUS performed upon intensive care unit admission, immediately following intubation, and upon clinical deterioration can provide valuable and timely clinical information to guide further therapy.

\section{Acknowledgment}

The authors would like to acknowledge the frontline HCWs in the New York Harbor Veterans Affairs system for their dedication in the care of our veterans and civilians in this time of crisis.

\section{Statement of Ethics}

Our research complies with the guidelines for human studies, and the research was conducted ethically in accordance with the World Medical Association Declaration of Helsinki.

Per recommendation of our Institutional Review Board, this case report was exempted from Institutional Review Board review. Informed consent was deemed unnecessary as personally identifiable information was removed.

\section{Disclosure Statement}

The authors have no conflicts of interest to declare.

\section{Funding Sources}

The authors report no funding source for this study.

\section{Author Contributions}

Dr. Lu Chen: case review and drafting of the manuscript. Dr. Gautham Upadhya: clinical care and editorial support. Dr. Uta Guo: editorial support. Dr. Pooja Belligund: clinical care and editorial support. Dr. David K. Lee: clinical care and editorial support. Dr. Isaac Shalom: clinical care and editorial support. Dr. Gangacharan R. Dubey: clinical care and editorial support. Dr. Mohammad R. Al-Ajam: clinical care and editorial support. Dr. Cristina A. Mitre: critical editing and concept development.

References

Cardiology 2020;145:467-472 DOI: $10.1159 / 000508527$
1 CDC. Coronavirus Disease 2019 (COVID-19) in the US [Internet]. Cent Dis Control Prev. 2020 Mar [cited 2020 Mar 29]. Available from: https://www.cdc.gov/coronavirus/2019. ncov/cases-updates/cases-in-us.html

2 Wang D, Hu B, Hu C, Zhu F, Liu X, Zhang J, et al. Clinical Characteristics of 138 Hospitalized Patients With 2019 Novel Coronavirus-Infected Pneumonia in Wuhan, China. JAMA. 2020 Feb;323(11):1061. 
3 Chen C, Chen C, Yan JT, Zhou N, Zhao JP, Wang DW. [Analysis of myocardial injury in patients with COVID-19 and association between concomitant cardiovascular diseases and severity of COVID-19]. Zhonghua Xin Xue Guan Bing Za Zhi. 2020 Mar;48(0):E008.

4 Shi S, Qin M, Shen B, Cai Y, Liu T, Yang F, et al. Association of cardiac injury with mortality in hospitalized patients with COVID-19 in Wuhan, China. JAMA Cardiol. 2020 Mar. DOI: 10.1001/jamacardio.2020.0950. Epub.

5 Li B, Yang J, Zhao F, Zhi L, Wang X, Liu L, et al. Prevalence and impact of cardiovascular metabolic diseases on COVID-19 in China. Clin Res Cardiol Off J Ger Card Soc. 2020 Mar. DOI: 10.1007/s00392-020-01626-9.

$6 \mathrm{Hu} \mathrm{H}, \mathrm{Ma} \mathrm{F}$, Wei X, Fang Y. Coronavirus fulminant myocarditis saved with glucocorticoid and human immunoglobulin. Eur Heart J. 2020 Mar. DOI: 10.1093/eurheartj/ehaa190. Epub.

7 Inciardi RM, Lupi L, Zaccone G, Italia L, Raffo $\mathrm{M}$, Tomasoni $\mathrm{D}$, et al. Cardiac involvement in a patient with coronavirus disease 2019 (COVID-19). JAMA Cardiol. 2020 Mar. DOI: 10.1001/jamacardio.2020.1096. Epub.

8 Huang C, Wang Y, Li X, Ren L, Zhao J, Hu Y, et al. Clinical features of patients infected with 2019 novel coronavirus in Wuhan, China. Lancet. 2020 Feb;395(10223):497-506.
9 Mégarbane B. Chloroquine and hydroxychloroquine to treat COVID-19: between hope and caution. Clin Toxicol (Phila). 2020 Apr. DOI: 10.1080/15563650.2020.1748194. Epub.

10 Ranney ML, Griffeth V, Jha AK. Critical Supply Shortages - The Need for Ventilators and Personal Protective Equipment during the Covid-19 Pandemic. N Engl J Med. 2020 Apr; 382(18):e41.

11 Nguyen T, Duong Bang D, Wolff A. 2019 novel coronavirus disease (COVID-19): paving the road for rapid detection and point-of-care diagnostics. Micromachines. 2020 Mar;11(3). DOI: $10.3390 / \mathrm{mil1030306.} \mathrm{Epub.}$

12 Buonsenso D, Piano A, Raffaelli F, Bonadia N, de Gaetano Donati K, Franceschi F. Point-ofCare Lung Ultrasound findings in novel coronavirus disease-19 pneumonia: a case report and potential applications during COVID-19 outbreak. Eur Rev Med Pharmacol Sci. 2020 Mar;24(5):2776-80.

13 Madjid M, Safavi-Naeini P, Solomon SD, Vardeny O. Potential effects of coronaviruses on the cardiovascular system: a review. JAMA Cardiol. 2020 Mar. DOI: 10.1001/jamacardio.2020.1286. Epub.
14 Tan ZC, Fu LH, Wang DD, Hong K. [Cardiac manifestations of patients with COVID-19 pneumonia and related treatment recommendations]. Zhonghua Xin Xue Guan Bing Za Zhi. 2020 Mar;48(0):E005.

15 Han H, Yang L, Liu R, Liu F, Wu KL, Li J, et al. Prominent changes in blood coagulation of patients with SARS-CoV-2 infection. Clin Chem Lab Med. 2020 Mar;0(0):/j/cclm. ahead-of-print/cclm-2020-0188/cclm-20200188.xml.

16 Li XY, Du B, Wang YS, Kang HY, Wang F, Sun B, et al. [The keypoints in treatment of the critical coronavirus disease 2019 patient]. Zhonghua Jie He He Hu Xi Za Zhi. 2020 Feb; 43(0):E026. Chinese.

17 Zhou F, Yu T, Du R, Fan G, Liu Y, Liu Z, et al. Clinical course and risk factors for mortality of adult inpatients with COVID-19 in Wuhan, China: a retrospective cohort study. Lancet. 2020 Mar;395(10229):1054-62.

18 Tang N, Bai H, Chen X, Gong J, Li D, Sun Z. Anticoagulant treatment is associated with decreased mortality in severe coronavirus disease 2019 patients with coagulopathy. J Thromb Haemost. 2020 May;18(5):1094-9.

19 Lai PS, Mita C, Thompson BT. What is the clinical significance of pulmonary hypertension in acute respiratory distress syndrome? A review. Minerva Anestesiol. 2014 May;80(5): $574-85$. 